\title{
Spreading the knowledge on the epidemiology of sepsis
}

Jorge I F Salluh, Marcio Soares, Mervyn Singer

Department of Critical Care, D'Or Institute for Research and Education, Rio de Janeiro CEP 22281-100, Brazil (JIFS, MSo); Internal Medicine Department, Universidade Federal do Rio de Janeiro, Rio de Janeiro, Brazil (JIFS, MSo); and Bloomsbury Institute of Intensive Care Medicine, Division of Medicine, University College London, London, UK (MSi) jorgesalluh@gmail.com

Notwithstanding the definition we choose, sepsis- associated short-term and long-term mortality and morbidity remain a major public health concern. From a high-income country perspective, the half-full glass (as shown by a sustained decline in mortality ${ }^{1,2}$ ) is counterbalanced by the half-empty glass in which the high burden of long-term cognitive and functional decline seen in survivors of long-term sepsis 3 is increasingly recognised. However, in low-income and middle-income countries, the growing evidence base obtained from recent epidemiological studies of sepsis leaves us far from optimistic. At a quick glance, the well-designed study by Flavia R Machado and colleagues ${ }^{4}$ shows that sepsis still has an ominous prognosis in patients admitted to a national-representative cohort of Brazilian intensive-care units (ICUs), with hospital mortality rates of 55\%. Even for basic therapies such as antimicrobials, there remains a large variation in availability, with many hospitals having suboptimal provision. In Brazilian ICU settings where good standards of care are provided, recent data show that sustained efforts to implement best practices with increased sepsis awareness are associated with decreased mortality rates and costs. 5,6 
There is no contradiction in these results. The contradiction lies in the heterogeneous access and quality of health care in low-income and middle-income countries. ${ }^{7}$ The excellent care provided in some hospitals in low-income and middle-income countries is in contrast with most, which frequently provide poor-quality care. Large urban populations usually have unsatisfactory primary care and have poor access to acute care despite the existence of a similar average number of ICU beds per person to European countries. ${ }^{7,8}$ Thus, the high burden of chronic diseases coupled with the inequality of public to private ICU bed ratio becomes an explosive combination. ${ }^{9}$

What else is behind the current numbers? A closer look at the SPREAD study ${ }^{4}$ reveals a mix of community- acquired and nosocomial sepsis that complicates identification of modifiable risk factors for mortality. ${ }^{4}$ When analysed separately, it becomes clear that outcome predictors in both groups are substantially different, as are the impact of process measures In combination and their association with sustained high mortality is key to implementing change (figure). This understanding of risk factors is essential, especially as they frequently represent opposing forces. So, an implementation of sepsis awareness and early treatment strategies might have its effects blunted by high rates of nosocomial infections or poor standards of care (eg, poor ventilator settings and sedation). ${ }^{10,11}$ 
In the SPREAD study, ${ }^{4}$ mortality rose progressively with a decreased availability of resources, and with inadequacy of treatment. Notably, no mortality difference was seen between private and public health-care systems, suggesting that payment for health care does not necessarily ensure better outcomes if quality of care remains suboptimal. Antibiotic compliance had no additional effect over compliance with a general 6-h bundle of care, suggesting that resource limitation in general might be a major factor in outcome determination. The nurse to patient ratio of 1:8 is much lower than nurse staffing levels in high-income countries. A high proportion (66\%) of the surveyed patients had sepsis acquired in hospital, with more than half of cases presenting in the ICU. This proportion of ICU-acquired sepsis is considerably higher than the approximately $10 \%$ rate reported in other studies such as the Sepsis-3 datasets from the USA. ${ }^{12}$ Infection control procedures are likely to be suboptimal in ICUs in low-income and middle-income countries. Better staffing levels and implementation of protocols which vary and trend towards low adherence to best practices. Understanding these factors individually and were associated with improved clinical outcomes in a retrospective cohort study involving 59693 patients in a large sample of Brazilian ICUs. ${ }^{13}$

These results from the study done by Machado and colleagues suggest that adoption of best practices are an efficient and practical means of improving outcomes and prevent ICU-acquired complications. One factor that was not captured in the SPREAD study 4 is the fact that triage procedures are virtually non-existent in daily Brazilian practice. There is ready access to intensive care for severely ill patients despite poor perceived outcomes and substantial comorbidities. Despite some improvements in regulations, cultural issues still limit the implementation of end of life care practices. Seen in this way, 
the explanations for the high mortality rates observed in septic patients in Brazil are easier to understand and, hopefully, offer potential targets for improvement that can be identified and tackled (figure). Studies in other countries could follow the path of the SPREAD study. Its design, aiming to be nationally representative, is both elegant and feasible, providing trustworthy information for clinicians,trialists, epidemiologists, and policy makers. Extrapolating incidence from a point prevalence study, however, might mean that the incidence is underestimated in the study by Machado and colleagues, given that patients with sepsis generally have much longer ICU stays. Further steps could also be taken to improve current knowledge and clinicians should not be deterred from asking uncomfortable questions. How should access be improved? What are the effects of socioeconomic status on sepsis? What are long- term outcomes in sepsis survivors? Rather than lumping all cases of sepsis together, community-acquired and hospital-acquired sepsis should perhaps be separated as different entities.

Traditional epidemiology can be advantageously merged with big data. Large databases are increasingly available in both high-income countries and low-income and middle-income countries. ${ }^{14}$ Studies in critical care $^{15,16}$ that made use of, for example, cluster analysis and machine learning techniques can unravel subgroups of critically-ill patients with distinct outcomes and responses to interventions. These approaches can open up new patterns and associations that were not previously considered using a traditional epidemiological approach. Inthe rightcontext, these insightsmight proveimportant targets for quality improvement initiatives. 


\section{References}

1 Kaukonen KM, Bailey M, Suzuki S, Pilcher D, Bellomo R. Mortality related to severe sepsis and septic shock among critically ill patients in Australia and New Zealand, 2000-2012. JAMA 2014; 311: 1308-16.

2 PRISM Investigators, Rowan KM, Angus DC, et al. Early, goal-directed therapy for septic shock-a patient-level meta-analysis. N Eng/ J Med 2017; 376: 2223-34.

3 Shankar-Hari M, Rubenfeld GD. Understanding long-term outcomes following sepsis: implications and challenges. Curr Infect Dis Rep 2016; 18: 37.

4 Machado FR, Cavalcanti AB, Bozza FA, et al, on behalf of the SPREAD Investigators and the Latin American Sepsis Institute Network. The epidemiology of sepsis in Brazilian intensive care units (the Sepsis PREvalence Assessment Database, SPREAD): an observational study. Lancet Infect Dis 2017; published online Aug 17. http://dx.doi.org/10.1016/ S1473-3099(17)30322-5.

5 Machado FR, Ferreira EM, Sousa JL, et al, for the Latin American Sepsis Institute Network. Quality improvement initiatives in sepsis in an emerging country: does the institution's main source of income influence the results? An analysis of 21,103 patients. Crit Care Med 2017; published online July 6. DOI: $10.1097 / C C M .0000000000002585$.

6 Noritomi DT, Ranzani OT, Monteiro MB, et al. Implementation of a multifaceted sepsis education program in an emerging country setting: clinical outcomes and cost-effectiveness in a long-term follow-up study. Intensive Care Med 2014; 40: 182-91.

7 Austin S, Murthy S, Wunsch $\mathrm{H}$, et al. Access to urban acute care services in high-vs middle-income countries: an analysis of seven cities. Intensive Care Med 2014; 40: 342-52. 
Bozza FA, Salluh Jl. An urban perspective on sepsis in developing countries. Lancet Infect Dis 2010;

10: $290-91$.

9 Rhodes A, Moreno RP. Intensive care provision: a global problem. Rev Bras Ter Intensiva 2012;

24: $322-25$.

Azevedo LC, Park M, Salluh JI, et al, for the ERICC (Epidemiology of Respiratory Insufficiency in Critical Care) investigators. Clinical outcomes of patients requiring ventilatory support in Brazilian intensive care units: a multicenter, prospective, cohort study. Crit Care 2013; 17: R63.

CHECKLIST-ICU Investigators and Brazilian Research in Intensive Care Network (BRICNet). Effect of a quality improvement intervention with daily round checklists, goal setting, and clinician prompting on mortality of critically ill patients: a randomized clinical trial. JAMA 2016; 315: 1480-90. Seymour CW, Liu VX, Iwashyna TJ, et al. Assessment of clinical criteria for sepsis: for the Third International Consensus Definitions for Sepsis and Septic Shock (Sepsis-3). JAMA 2016; 315: 762-74. Soares M, Bozza FA, Angus DC, et al. Organizational characteristics, outcomes, and resource use in 78 Brazilian intensive care units: the ORCHESTRA study. Intensive Care Med 2015; 41: 2149-60. Iwashyna TJ, Liu V. What's so different about big data? A primer for clinicians trained to think epidemiologically. Ann Am Thorac Soc 2014; 11: 1130-35.

15 Vranas KC, Jopling JK, Sweeney TE, et al. Identifying distinct subgroups of ICU patients: a machine learning approach. Crit Care Med 2017; published June 21. DOI: 10.1097/CCM.0000000000002548. Famous KR, Delucchi K, Ware LB, et al, for the ARDS Network. Acute respiratory distress syndrome subphenotypes respond differently to randomized fluid management strategy. Am J Respir Crit Care Med 2017; 195: 331-38. 
Figure: Factors contributing to sustained high mortality in community-acquired sepsis in lowincome and middle-income countries: a roadmap for future studies?

$E O L=e n d$ of life. $I C U=$ intensive-care unit. $\mathrm{HAI}=$ health-care-associated infections
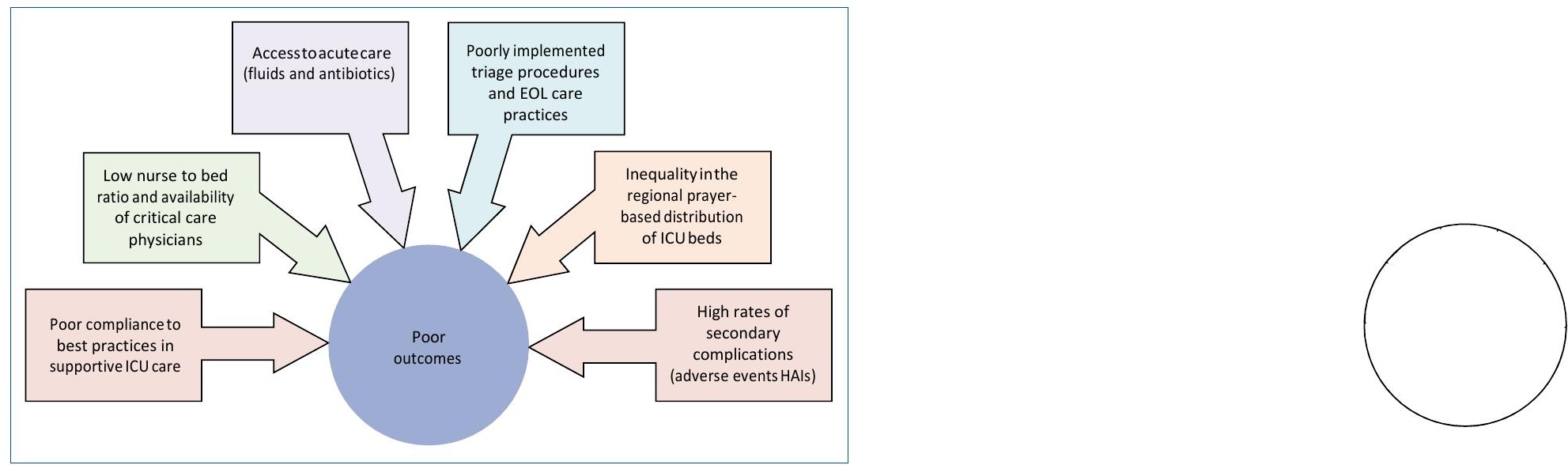